\title{
Emerging Role of Pharmacogenetic in Organ Transplantation
}

\author{
Mariano Ferraresso*
}

Division of General and Vascular Surgery, Saint Joseph Hospital-IRCCS Multimedica, Milan, Italy

Department of Surgical Science, University of Milan, Italy

\begin{abstract}
The currently used immunosuppressive drugs have a narrow therapeutic index which required to individualize the dose regimen for different recipients. Pharmacogenetic is the use of genetic screening to prevent metabolic responses to different immunosuppressive drugs. Since the oxidative enzymes cytochrome P450 CYP3A and the drug efflux pump P-glycoprotein (P-gp) play a pivotal role in immunosuppressive drugs metabolism, pharmacogenetic studies have been mainly focused on these two enzymes. This would provide an important aid toward drug regimen individualization during the post-transplant therapy and has potential to improve graft outcome.
\end{abstract}

Keywords: Pharmacogenetics; Calcineurine inhibitors; Organ transplantation; CYP3A5; $A B C B 1$

\section{Introduction}

Organ transplantation is becoming the optimal therapy strategy for end stage renal, liver heart and lung diseases. The introduction of better and more specific immunosuppressive drugs have enable organ replacement to obtain its current place. In fact, a gradual improvement in short-term graft survival has occurred over the last 10 years and extensive use of the calcineurine inhibitors (CI) tacrolimus and cyclosporine, have played a not negligible role [1]. However, there has been minimal improvement in long term graft survival over the same period partly due to drug side effects [2,3]. It is well recognized that response to $\mathrm{CI}$ has significant inter and intra individual variation in transplant patients. Both over and under-dosage of CI influence the life span of the graft and of the recipient [4,5]. Several factors such as organ function, drug interaction and the nature of the disease may influence the effects of CI. Recently, the role of genetic factors has been also pointed out since genetic polymorphisms are implicated in the interindividual variability of the pharmacokinetic and pharmacodynamic characteristics of many drugs. Pharmacogenetic is the study of genetic variations that give rise to different drug responses and theoretically represents a very promising tool. A genetic screening prior to transplantation should allow to forecast the individual response to a given immunosuppressive drug therefore to tailor immunosuppressive regimen in order to optimized short and long term outcomes.

\section{Metabolic Pathways as Possible Targets for a Pharmacogenetic Strategy}

The genes that are primarily involved in metabolizing immunosuppressant are those encoding cytochrome P450 (CYP) $3 \mathrm{~A}$ family enzymes and multidrug resistance P-glycoprotein 1 (the $A B C B 1$ gene). As many drugs undergo substantial intestinal and liver metabolism after absorption from the gut lumen, it is believed that CYP3A and P-glycoproteinare are lagely responsible for the poor oral bioavailability of calcineurin inhibitors [6]. The CYP3A subfamily is a cluster consisting of four isoenzymes. CYP3A4 and CYP3A5 are the main genes involved in the metabolism of cyclosporine and tacrolimus [7]. CYP3A5 is expressed more variably and at a relatively higher level in the proximal renal tubules [8]. CYP3A5*1 encodes an active protein and is responsible for the increased metabolism of many drugs including tacrolimus but not cyclosporine [6]. During the transcription phase, the CYP3A5*3 (6986 A>G) allele introduces an alternative splicing site leading to protein truncation, thus resulting in the absence of full-length protein expression $[9,10]$. CYP3A4 is abundantly and constitutively expressed in hepatic and intestinal epithelia, so the reported increase in transcriptional activity shown by the CYP3A4*1B allele in vitro would theoretically also reflect significant enzymatic activity in vivo [11]. P-gp, which is encoded by the $A B C B 1$ gene, is a large ATP-dependent transmembrane protein involved in the extracellular extrusion of many xenobiotics and drugs including calcineurin inhibitors (CNIs) [12]. Cyclosporine is a typical $A B C B 1$ substrate and intestinal P-gp may be responsible for the reduced intestinal absorption of cyclosporine [13]. More than 700 variations in the nucleotide sequences have been described and although nothing is known about the clinical impact of most of the sequence variations on P-gp function, some seem to be functionally relevant and substantially influence the pharmacokinetics of substrate drugs. The most extensively investigated SNPs of $A B C B 1$ are $3435 \mathrm{C}>$ $\mathrm{T}(\mathrm{rs} 1045642)$ in exon 26, 1236C > T (rs 128503) in exon 12 and $2677 \mathrm{G}$ $>\mathrm{T} / \mathrm{A}$ (rs 2032582) in exon 21 [14]. The 3435C > T polymorphism is a silent polymorphism that may be in linkage disequilibrium with other functional polymorphisms in the $A B C B 1$ gene, including $2677 \mathrm{G}$ > T/A. The $3435 \mathrm{C}>\mathrm{T}$ polymorphism may also reduce $A B C B 1 \mathrm{mRNA}$ stability in the liver and it could change protein folding and activity [15]. Another interesting SNP is IVS21+49 T > C (rs2032583) in intron 21 of $A B C B 1$, which is involved in the pharmacokinetics of many drugs including antidepressants and methadone [16].

\section{CYP3A5 and ABCB1 Genotypes and Tacrolimus}

The expression of CYP3A5 is associated with reduced tacrolimus exposure following oral administration in both pediatric and adult population [17,18]. CYP3A5 expressors exhibit a significant delay in achieving target blood concentration [19]. Therefore, an increased incidence of acute rejection episodes in the early post-transplant period has been reported [20]. Recently, the first study to show the prospective adaptation of tacrolimus daily dose based on CYP3A5 patient's genotype, confirmed that pre-transplant genetic screening increased significantly the proportion of patients reaching the therapeutic target range. In fact, in this multi-center randomized controlled trial, patients receiving tacrolimus daily dose according to their CYP3A5 genotype,

${ }^{*}$ Corresponding author: Mariano Ferraresso, M.D. Ph.D., Department of Surgical Science, University of Milan, Via San Vittore 12, 20122 Mila, Italy, Tel: +39 0250320382; Fax: +39 0250320384; E-mail: Mariano.ferraresso@unimi.it

Received July 03, 2020; Accepted July 06, 2012; Published July 10,2020

Citation: Ferraresso M (2020) Emerging Role of Pharmacogenetic in Organ Transplantation. J Transplant Technol Res 2:e110. doi:10.4172/21610991.1000e110

Copyright: (C) 2020 Ferraresso M. This is an open-access article distributed under the terms of the Creative Commons Attribution License, which permits unrestricted use, distribution, and reproduction in any medium, provided the original author and source are credited. 
achieved the target concentration more rapidly with lesser dose modification and in an higher percentage $(>75 \%)$ when compared to those in whom tacrolimus was managed with a concentrationcontrol strategy [21]. ABCB1 polymorphisms do not seem to influence tacrolimus pharmacokinetic and research into this association has yielded mixed results $[22,23]$. Nevertheless, in our series we observed a significant reduction in tacrolimus concentration and dose-adjusted tacrolimus concentrations in the early post-transplant period for the TT polymorphism of C1236T; this suggests that there is higher metabolis $m$ of the drug in subjects with this genotype [24]. This could be substantiated by the fact that 1236 and 3435 polymorphisms of $A B C B 1$ influence the shaping and stability of mRNA and, consequently, gene expression [25]. Interestingly, a recent study on pediatric liver transplantation demonstrated a significant association between $A B C B 1$ polymorphisms and tacrolimus-related nephrotoxicity [26]. This suggests that $A B C B 1$ polymorphisms in the gastrointestinal tract do have an influence on tacrolimus dose requirement and exposure.

\section{CYP3A5 and ABCB1 Genotypes and Cyclosporine}

There are a number of conflicting reports regarding the CYP3A genotype influence on cyclosporine pharmacokinetic [27,28]. Therefore, a clear role of this polymorphism on cyclosporine metabolism has not been demonstrated. However, it is necessary to bear in mind that the drug is simultaneously both a substrate and an inhibitor of $A B C B 1$ [29] which is why the influence of $A B C B 1$ polymorphisms is rather controversial. In our series, we found that $A B C B 1$ polymorphisms affected cyclosporine pharmacokinetics in the immediate post-transplant period rather later [24]. The time-related effect of $A B C B 1$ polymorphisms may be explained by the fact that the drug's potent inhibitory effect on P-gp function requires an adequate pharmacological load before it is fully expressed [30]. Another possible explanation should be related to the age of the recipients. Recently, Fanta and colleagues suggested that age-related polymorphisms of $A B C B 1$ could explain the different oral bioavailability of cyclosporine among different age groups [31].

\section{The Effect of Donor Genetic Polymorphisms on the Transplant Outcome}

Among the genetic variables that might affect immunosuppressive drug metabolism, consideration should be given to the possible role of donor genotype. In fact, grafts from donors may carry different genotypes from CYP3A5 and $A B C B 1$. However very few data are available on whether and how much the donor graft genetic background influence absorption and metabolism of immunosuppressive drugs. This would mainly apply to liver transplantation where particular receptors in the donor's graft have been reported to substantially affect tacrolimus metabolism $[32,33]$. In the kidney transplant setting there have been relatively few studies looking at the role of polymorphisms in graft donors and their conclusions remains unclear. Woillard et al. [34] reported that the presence of $A B C B 1$ polymorphisms in donors influenced long-term graft outcome of renal transplant patients. Nevertheless, Hauser et al. [35] reported that in adult renal transplant recipients the incidence of cyclosporine nephrotoxicity was significantly higher when the donor rather than the recipient, had the $A B C B 13435 T T$ genotype. Obviously, intra-graft $A B C B 1$ expression is related to donor's genotype and possibly responsible for cyclosporine accumulation in renal tubular cells. Further studies are required to evaluate the role of donor polymorphisms although it is likely that in pediatric and adult renal transplantation the role of recipient polymorphisms will be more important.

\section{Conclusions}

In organ transplantation the poor bioavailability and the large intra and inter-individual variability in the administration of immunosuppressive drugs limit the postoperative drug therapy, which may subsequently affect the function and lifespan of grafts. The individualization of the therapeutic regimens in different patients is mandatory in order to balance clinical efficacy and toxicity. CYP3A and P-gp are the two key enzymes which greatly influence the absorption, disposition, metabolism and clearance of immunosuppressive drugs. Many genetic factors have been identified to affect the expression of CYP3A and P-gp and up to day there is a large evidence base relating to genetic influences on the pharmacology of calcineurine inhibitors. The pre-transplant screening of the most relevant polymorphisms should therefore be considered in order to tailor immunosuppressive therapy to an individual patient's metabolism.

\section{References}

1. Halloran PF (2004) Immunosuppressive drugs for kidney transplantation. N Engl J Med 351: 2715-2729.

2. Golshayan D, Buhler L, Lechler RI, Pascual M (2007) From current immunosuppressive strategies to clinical tolerance of allografts. Transpl Int 20: 12-24.

3. Kaneku HK, Terasaki PI (2006) Thirty year trend in kidney transplants: UCLA and UNOS Renal Transplant Registry. Clin Transpl 1-27.

4. Mueller EA, Kovarik JM, van Bree JB, Lison AE, Kutz K (1994) Pharmacokinetics and tolerability of a microemulsion formulation of cyclosporine in renal allograft recipients--a concentration-controlled comparison with the commercial formulation. Transplantation 57: 1178-1182.

5. Venkataramanan R, Swaminathan A, Prasad T, Jain A, Zuckerman S, et al. (1995) Clinical pharmacokinetics of tacrolimus. Clin Pharmacokinet 29: 404430.

6. Hesselink DA, van Schaik $\mathrm{RH}$, van der Heiden $\mathrm{IP}$, van der Werf $\mathrm{M}$, Gregoor PJ, et al. (2003) Genetic polymorphisms of the CYP3A4, CYP3A5, and MDR1 genes and pharmacokinetics of the calcineurin inhibitors cyclosporine and tacrolimus. Clin Pharmacol Ther 74: 245-254.

7. Kronbach T, Fischer V, Meyer UA (1988) Cyclosporine metabolism in human liver: identification of a cytochrome P-450III gene family as the major cyclosporine-metabolizing enzyme explains interactions of cyclosporine with other drugs. Clin Pharmacol Ther 43: 630-635.

8. Cummings BS, Parker JC, Lash LH (2000) Role of cytochrome P450 and glutathione S-transferase alpha in the metabolism and cytotoxicity of trichloroethylene in rat kidney. Biochem Pharmacol 59: 531-543.

9. Hustert E, Haberl M, Burk O, Wolbold R, He YQ, et al. (2001) The genetic determinants of the CYP3A5 polymorphism. Pharmacogenetics 11: 773-779.

10. Kuehl P, Zhang J, Lin Y, Lamba J, Assem M, et al. (2001) Sequence diversity in CYP3A promoters and characterization of the genetic basis of polymorphic CYP3A5 expression. Nat Genet 27: 383-391.

11. Amirimani B, Walker AH, Weber BL, Rebbeck TR (1999) Modification of clinical presentation of prostate tumors by a novel genetic variant in CYP3A4. J Natl Cancer Inst 91: 1588-1590.

12. Leonard GD, Fojo T, Bates SE (2003) The role of ABC transporters in clinical practice. Oncologist 8: 411-424.

13. Lown KS, Mayo RR, Leichtman AB, Hsiao HL, Turgeon DK, et al. (1997) Role of intestinal P-glycoprotein (mdr1) in interpatient variation in the oral bioavailability of cyclosporine. Clin Pharmacol Ther 62: 248-260.

14. Hoffmeyer S, Burk O, von Richter O, Arnold HP, Brockmöller J, et al. (2000) Functional polymorphisms of the human multidrug-resistance gene: multiple sequence variations and correlation of one allele with P-glycoprotein expression and activity in vivo. Proc Natl Acad Sci U S A 97: 3473-3478.

15. Komar AA (2007) Silent SNPs: impact on gene function and phenotype. Pharmacogenomics 8: 1075-1080.

16. Levran O, O'Hara K, Peles E, Li D, Barral S, et al. (2008) ABCB1 (MDR1) genetic variants are associated with methadone doses required for effective treatment of heroin dependence. Hum Mol Genet 17: 2219-2227. 
Citation: Ferraresso M (2020) Emerging Role of Pharmacogenetic in Organ Transplantation. J Transplant Technol Res 2:e110. doi: 10.4172/2161$0991.1000 \mathrm{e} 110$

Page 3 of 3

17. MacPhee IA, Fredericks S, Tai T, Syrris P, Carter ND, et al. (2004) The influence of pharmacogenetics on the time to achieve target tacrolimus concentrations after kidney transplantation. Am J Transplant 4: 914-919.

18. Ferraresso M, Tirelli A, Ghio L, Grillo P, Martina V, et al. (2007) Influence of the CYP3A5 genotype on tacrolimus pharmacokinetics and pharmacodynamics in young kidney transplant recipients. Pediatr Transplant 11: 296-300.

19. Macphee IA, Fredericks S, Mohamed M, Moreton M, Carter ND, et al. (2005) Tacrolimus pharmacogenetics: the CYP3A5*1 allele predicts low dosenormalized tacrolimus blood concentrations in whites and South Asians. Transplantation 79: 499-502.

20. Quteineh L, Verstuyft C, Furlan V, Durrbach A, Letierce A, et al. (2008) Influence of CYP3A5 genetic polymorphism on tacrolimus daily dose requirements and acute rejection in renal graft recipients. Basic Clin Pharmacol Toxicol 103: 546552.

21. ThervetE, Loriot MA, Barbier S, Buchler M, Ficheux M, et al. (2010) Optimization of initial tacrolimus dose using pharmacogenetic testing. Clin Pharmacol Ther 87: $721-726$

22. Kuypers DR, de Jonge H, Naesens M, Lerut E, Verbeke K, et al. (2007) CYP3A5 and CYP3A4 but not MDR1 single-nucleotide polymorphisms determine longterm tacrolimus disposition and drug-related nephrotoxicity in renal recipients. Clin Pharmacol Ther 82: 711-725.

23. Press RR, Ploeger BA, den Hartigh J, van der Straaten T, van Pelt J, et al. (2009) Explaining variability in tacrolimus pharmacokinetics to optimize early exposure in adult kidney transplant recipients. Ther Drug Monit 31:187-197.

24. Turolo S, Tirelli AS, Ferraresso M, Ghio L, Belingheri M, et al. (2010) Frequencies and roles of CYP3A5, CYP $3 A 4$ and $A B C B 1$ single nucleotide polymorphisms in Italian teenagers after kidney transplantation. Pharmaco Rep 62: 1159-1169.

25. Wang J, Zeevi A, McCurry K, Schuetz E, Zheng H, et al. (2006) Impact of ABCB1 (MDR1) haplotypes on tacrolimus dosing in adult lung transplant patients who are $C Y P 3 A 5^{\star} 3{ }^{\star} 3$ non-expressors. Transpl Immunol 15: 235-240.

26. Hawwa AF, McKiernan PJ, Shields M, Millership JS, Collier PS, et al. (2009) Influence of $A B C B 1$ polymorphisms and haplotypes on tacrolimus nephrotoxicity and dosage requirements in children with liver transplant. $\mathrm{Br} \mathrm{J}$ Clin Pharmacol 68: 413-421.

27. Kreutz R, Zuurman M, Kain S, Bolbrinker J, de Jong PE, et al. (2005) The role of the cytochrome P450 3A5 enzyme for blood pressure regulation in the general Caucasian population. Pharmacogenet Genomics 15:831-837.

28. Fredericks S, Jorga A, MacPhee IA, Reboux S, Shiferaw E, et al. (2007) Multidrug resistance gene-1 (MDR-1) haplotypes and the CYP3A5*1 genotype have no influence on ciclosporin dose requirements as assessed by $\mathrm{C} 0$ or $\mathrm{C} 2$ measurements. Clin Transplant 21: 252-257.

29. Bartlett NL, Lum BL, Fisher GA, Brophy NA, Ehsan MN, et al. (1994) Phase I trial of doxorubicin with cyclosporine as a modulator of multidrug resistance. Clin Oncol 12: 835-842.

30. van Gelder T, Klupp J, Sawamoto T, Christians U, Morris RE (2001) ATPbinding cassette transporters and calcineurin inhibitors: potential clinical implications. Transplant Proc 33: 2420-2421.

31. Fanta S, Niemi M, Jönsson S, Karlsson MO, Holmberg C, Neuvonen PJ, Hoppu $\mathrm{K}$, Backman JT (2008) Pharmacogenetics of cyclosporine in children suggests an age-dependent influence of $A B C B 1$ polymorphisms. Pharmacogenet Genomics 18: 77-90.

32. Yu S, Wu L, Jin J, Yan S, Jiang G, et al. (2006) Influence of CYP3A5 gene polymorphisms of donor rather than recipient to tacrolimus individual dose requirement in liver transplantation. Transplantation 81: 46-51.

33. Uesugi M, Masuda S, Katsura T, Oike F, Takada Y, et al. (2006) Effect of intestinal CYP3A5 on postoperative tacrolimus trough levels in living-donor liver transplant recipients. Pharmacogenet Genomics 16: 119-127.

34. Woillard JB, Rerolle JP, Picard N, Rousseau A, Guillaudeau A, et al. (2010) Donor P-gp polymorphisms strongly influence renal function and graft loss in a cohort of renal transplant recipients on cyclosporine therapy in a long-term follow-up. Clin Pharmacol Ther 88: 95-100.

35. Hauser IA, Schaeffeler E, Gauer S, Scheuermann EH, Wegner B, et al. (2005) $A B C B 1$ genotype of the donor but not of the recipient is a major risk factor for cyclosporine-related nephrotoxicity after renal transplantation. J Am Soc Nephrol 16: 1501-1511. 